\title{
Matching covered graphs with three removable classes
}

\author{
Marcelo H. de Carvalho* \\ FACOM-UFMS \\ Campo Grande \\ Brazil
}

mhc@facom.ufms.br

\author{
C. H. C. Little \\ Massey University \\ Palmerston North \\ New Zealand
}

C.Little@massey.ac.nz

Submitted: Aug 24, 2012; Accepted: Apr 10, 2014; Published: May 2, 2014

Mathematics Subject Classifications: 05C70, 05C75

\begin{abstract}
The notion of removable classes arises in connection with ear decompositions of matching covered graphs introduced by Lovász and Plummer. The last (single or double) ear of an ear decomposition is defined as a removable class. Every matching covered graph not induced by a circuit has at least three removable classes. In this paper, we characterize matching covered graphs with precisely three removable classes and show, as a corollary, that every non-planar matching covered graph has at least four removable classes. Let $G$ be a matching covered graph. A matching covered subgraph $H$ of $G$ is conformal if $G-V H$ has a perfect matching. Given $S \subseteq E G$, what is a minimal conformal subgraph of $G$ that contains $S$ ? It is known that if $|S|=2$ then it is induced by a circuit. As an application of the main result, we answer this question for $|S|=3$.
\end{abstract}

Keywords: graph theory; perfect matchings; matching covered graphs

\section{Matching covered graphs}

The graphs considered here are loopless, but they may have multiple edges. The notation and terminology we use is essentially that of Bondy and Murty [1].

A connected graph $G$ is matching covered if each of its edges lies in a perfect matching. Some authors refer to matching covered graphs as 1-extendable graphs. The treatise by Lovász and Plummer [11] and the seminal work by Lovász [10] on the matching lattice contain the basic theory of matching covered graphs. For the convenience of the reader, we shall briefly review here the terminology and results which are pertinent to this article.

\footnotetext{
${ }^{*}$ Supported by Fundect-MS and CNPq, Brazil.
} 


\subsection{Conformal subgraphs}

Let $G$ be a matching covered graph. A matching covered subgraph $H$ of $G$ is conformal if $G-V H$ has a perfect matching.

An even subdivision of a graph $G$ is a graph obtained from $G$ by replacing each edge $e$ with an odd path joining the ends of $e$ but having none of its internal vertices in $V G$. Thus, any graph is an even subdivision of itself. It is easy to see that a graph $G$ is matching covered if and only if every even subdivision of $G$ is matching covered. The following is a classical result due to Lovász.

Theorem 1. ([9]) Every nonbipartite matching covered graph contains a conformal subgraph that is an even subdivision of $K_{4}$ or $\overline{C_{6}}$.

The following result plays an important role in the proof of our main result.

Theorem 2. ([2]) Any set of $k \geqslant 2$ edges of a matching covered graph $G$ lies in a conformal subgraph formed by the union of at most $k-1 M$-alternating circuits, for some perfect matching $M$ of $G$.

It follows from the theorem above that any two edges of a matching covered graph lie in a conformal circuit. (See also [8].) This paper extends this result to three edges. More precisely, given a matching covered graph $G$ and a set $S$ of three edges of $G$, we characterize minimal conformal subgraphs of $G$ that contain the edges of $S$.

\subsection{Cuts, contractions and splicings}

Let $G$ be a connected graph. For any set $X$ of vertices of $G$, we denote the coboundary of $X$ by $\partial_{G} X$. Thus, $\partial_{G} X$ consists precisely of those edges that have one end in $X$ and one end in the complement $\bar{X}$ of $X$. If $G$ is understood, we write simply $\partial X$ instead of $\partial_{G} X$. The set $\partial X$ is called a cut and the sets $X$ and $\bar{X}$ are its shores. A cut is odd if both its shores have an odd number of vertices and is trivial if one of its shores is a singleton.

Given a cut $C=\partial X$ of $G$, where $X$ is a nonempty proper subset of $V G$, the two graphs obtained by contracting $X$ to a single vertex $x$ and $\bar{X}$ to a single vertex $\bar{x}$ are called the $C$-contractions of $G$. A graph $G$ is the splicing of two graphs $G_{1}$ and $G_{2}$ if it has a cut $C$ such that $G_{1}$ and $G_{2}$ are isomorphic to the two $C$-contractions of $G$. The following assertion may be easily verified.

Proposition 3. Any splicing of two matching covered graphs is also matching covered.

\subsection{Separating and tight cuts}

A cut $C$ in a matching covered graph $G$ is separating if both $C$-contractions are matching covered. Each shore of $C$ then induces a connected subgraph of $G$. The following result is deduced directly from this definition.

Proposition 4. Let $G$ be a matching covered graph. A cut $C$ of $G$ is separating if and only if every edge of $G$ lies in a perfect matching that contains precisely one edge in $C$. 
A separating cut $C$ of a matching covered graph $G$ is tight if $|M \cap C|=1$ for every perfect matching $M$ of $G$. Thus, every tight cut is also separating. The converse does not hold in general. For example, the graph $\overline{C_{6}}$ and the Petersen graph have separating cuts that are not tight.

A matching covered graph is solid if each separating cut is tight. Every bipartite matching covered graph is solid. Odd wheels and Möbius ladders are also examples of solid matching covered graphs [7]. In fact, these graphs have no separating cut. The Petersen graph and $\overline{C_{6}}$ are examples of nonsolid matching covered graphs.

A matching covered graph free of nontrivial tight cuts is called a brace if it is bipartite, a brick otherwise.

\subsection{Ear decompositions}

An ear in a matching covered graph $G$ is a path $P$ of odd length such that both ends of $P$ have degree at least 3 in $G$ but all the internal vertices of $P$ have degree 2 in $G$. For an ear $P$, the graph $G-P$ is the graph obtained from $G$ by deleting all edges and internal vertices of $P$, and $P$ is said to be removable if $G-P$ is matching covered. A double ear in $G$ is a pair $\left\{P_{1}, P_{2}\right\}$ of vertex-disjoint ears. A double ear $\left\{P_{1}, P_{2}\right\}$ is removable if neither $P_{1}$ nor $P_{2}$ is removable, but $G-\left(P_{1} \cup P_{2}\right)=\left(G-P_{1}\right)-P_{2}$ is matching covered. The following theorem is one of the basic results of the theory of matching covered graphs $[11$, Chapter 5].

Theorem 5 (Ear decomposition). Let $G$ be a matching covered graph not induced by a circuit, and let $H$ be a conformal matching covered subgraph of $G$. Then there exists a sequence $\left(G_{1}, G_{2}, \ldots, G_{r}\right)$ of subgraphs of $G$ such that $G_{1}=G, G_{r}=H$ and, for $2 \leqslant i \leqslant r$, $G_{i}$ is obtained from $G_{i-1}$ by deleting either a removable ear or a removable double ear of $G_{i-1}$.

A single removable ear, or the pair of ears that constitute a removable double ear, will be referred to as a removable class. An edge $e$ is also described as removable if $G-\{e\}$ is matching covered. The following results present basic properties of removable classes.

Lemma 6. The removable classes of a matching covered graph not induced by a circuit are pairwise disjoint.

Proof: Let $G$ be a matching covered graph not induced by a circuit. Let $R$ be a removable class of $G$ and let $e$ be any edge of $R$. Let us show that $R$ is the only removable class that contains $e$.

As $G$ is not induced by a circuit, $R$ includes a unique ear $P$ that contains $e$. If $R$ is a single ear then $R=\{P\}$ and $R$ is the only removable class that contains $e$. Suppose therefore that $R$ is a double ear. Then there must be another ear $Q$ such that $R=\{P, Q\}$. Moreover, $G-P$ is connected (because $G$ is 2-connected) but not matching covered, and so there exists an edge of $E G-P$ not in any 1 -factor of $G-P$. This edge must be in $Q$ since $G-(P \cup Q)$ is matching covered, and so $Q$ is uniquely determined. Hence $e$ belongs to a unique removable class. 
Corollary 7. Every matching covered graph not induced by a circuit has at least three removable classes.

Proof: Let $G$ be a matching covered graph not induced by a circuit. Consider an ear decomposition $\left(G_{1}, G_{2}, \ldots, G_{r}\right)$ of $G$. By Theorem $5, G_{2}$ is obtained from $G$ by deleting a removable class, say $Q_{1}$, of $G$.

Now consider an alternating circuit $C$ containing an edge of $Q_{1}$. We claim that $Q_{1} \subset C$. This is clearly true if $Q_{1}$ is a single ear. If $Q_{1}$ is a double ear $\left\{Q_{1}^{\prime}, Q_{1}^{\prime \prime}\right\}$ and $C$ contains $Q_{1}^{\prime}$ but not $Q_{1}^{\prime \prime}$ then $Q_{1}^{\prime \prime}$ would be a removable single ear of $G$ (because $G\left[E G_{2} \cup C\right]$ is matching covered), in contradiction to the definition of a removable double ear. As asserted, $Q_{1} \subset C$. Now, applying the ear decomposition theorem with $H=C$, we get a second removable class $Q_{2}$ of $G$.

By Theorem 2, there exists an alternating circuit $D$ containing an edge of $Q_{1}$ and an edge of $Q_{2}$. By the same reasoning as above, we have $Q_{1} \cup Q_{2} \subset D$. By applying the ear decomposition theorem again with $H=D$, we get a third removable class of $G$.

An attempt to continue the reasoning in this proof to find a fourth removable class fails because the "new" removable class is not necessarily distinct from the three already found.

\subsection{A dependence relation}

Let $G$ be a matching covered graph, and let $e$ and $f$ be any two edges of $G$. Then $e$ depends on $f$, or $e$ implies $f$, if every perfect matching that contains $e$ also contains $f$. We write $e \Rightarrow f$ to indicate that $e$ depends on $f$. We say that two edges $e$ and $f$ are mutually dependent if $e \Rightarrow f$ and $f \Rightarrow e$. In this case we write $e \Leftrightarrow f$. Clearly $\Leftrightarrow$ is an equivalence relation.

A set $Q$ of mutually dependent edges of $G$ such that no edge in $E G-Q$ depends on an edge of $Q$ is called a minimal class of $G$. Each minimal class therefore consists of a set of independent edges, and distinct minimal classes are disjoint. For instance, a removable singleton or doubleton of a matching covered graph is a minimal class. Let $e$ be an edge of $G$. Any minimal class $Q$ that contains an edge that depends on $e$ is said to be a minimal class induced by $e$. In this case every edge of $Q$ depends on $e$. Minimal classes in a brick have some attractive properties.

Theorem 8. ([10, Lemma 3.4]) Let $G$ be a brick and $Q$ a minimal class of $G$. Then $|Q| \leqslant 2$. Moreover, if $|Q|=2$, then $G-Q$ is bipartite.

\section{Extremal graphs and their properties}

A matching covered graph not induced by a circuit is extremal if it has precisely three removable classes.

It is easy to see that forming an even subdivision does not change the number of removable classes of a matching covered graph. The following result summarizes this property. 
Proposition 9. A graph $G$ is extremal if and only if every even subdivision of $G$ is extremal.

The next result provides a more precise idea of an extremal graph.

Lemma 10. An extremal graph is an even subdivision of a cubic graph.

Proof: Let $G$ be an extremal graph. Let $Q_{1}, Q_{2}$ and $Q_{3}$ be the three removable classes of $G$. Let $e_{i}, i=1,2,3$, be an edge of $Q_{i}$.

By Theorem 2, $G$ has a 2-connected conformal subgraph containing $e_{1}, e_{2}$ and $e_{3}$ formed by the union of two $M$-alternating circuits $C$ and $D$, for some perfect matching $M$ of $G$. Then, $H=C \cup D$ contains $Q_{1}, Q_{2}$ and $Q_{3}$. If $G$ is distinct from $H$ then, by considering an ear decomposition of $G$ finishing with $H$, we find that the first removable class of $G$ is distinct from $Q_{1}, Q_{2}$ and $Q_{3}$, which is a contradiction. Thus, $G=H$.

As circuits $C$ and $D$ are alternating with the same perfect matching $M$, each vertex common to $C$ and $D$ is incident with an edge that is also common to $C$ and $D$. It follows that $G$ is an even subdivision of a cubic graph.

In view of Proposition 9 and Lemma 10, to characterize extremal graphs, it suffices to characterize cubic extremal graphs. Thus, we turn our attention to characterizing cubic extremal graphs. Let $\Theta$ be the graph with just two vertices and three links joining them.

Proposition 11. Every cubic extremal graph is simple, unless it is the $\Theta$ graph.

Proof: Let $G$ be a cubic extremal graph, and let $e$ and $f$ be parallel edges of $G$. Then, $G-\{e\}$ and $G-\{f\}$ are matching covered. Then $\{\{e\}\}$ is a removable class of $G$. Analogously, $\{\{f\}\}$ is a removable class of $G$.

If $G-\{e\}$ is not a circuit then, by Corollary $7, G-\{e\}$ has at least three removable classes. At most one removable class of $G-\{e\}$ contains $\{f\}$. The removable classes of $G-\{e\}$ that do not contain $\{f\}$ are removable classes of $G$. Then, $G$ has at least four removable classes, a contradiction.

Thus, $G-\{e\}$ is a circuit. Then, $G$ is a subdivision of $\Theta$. As $G$ is cubic, it follows that $G$ is, in fact, the $\Theta$ graph.

\section{$3 \quad$ Extremal graphs free of separating cuts}

As every tight cut is also separating, an extremal graph free of separating cuts is also free of tight cuts, that is, it is a brick or a brace. By Lemma 10, it is cubic. Thus, in this section, we characterize cubic extremal bricks and braces free of separating cuts. We shall make use of an important property concerning removable edges in braces.

Lemma 12. ([5, Lemma 3.2]) Let $G$ be a brace on at least six vertices. Then every edge of $G$ is removable.

The following result characterizes cubic extremal braces. 
Theorem 13. The only cubic extremal brace is the $\Theta$ graph.

Proof: By Lemma 12, every edge of a brace on six or more vertices is removable. A brace on six or more vertices has at least nine edges. Thus, an extremal brace has at most four vertices. But, there is only one connected cubic bipartite graph on four vertices and it has four removable edges. Therefore, a cubic extremal brace has two vertices. The only cubic extremal graph on two vertices is the $\Theta$ graph.

We now proceed to characterize extremal bricks free of separating cuts. Recall that a brick free of separating cuts is a solid brick. We shall show that $K_{4}$ is the only extremal solid brick. The following result will be used.

Lemma 14. ([4, Corollary 6.15]) If $G$ is a solid brick of maximum degree 3 or 4, then, for every vertex $v$ of $G$, at most one edge incident with $v$ does not lie in a removable class of $G$.

Theorem 15. Graph $K_{4}$ is the only solid extremal brick.

Proof: Let $G$ be a solid extremal brick. Let $H$ be the subgraph of $G$ spanned by the union of the removable classes of $G$. By Lemma 14, $H$ has minimum degree at least 2 and spans $G$. By Theorem 8, every removable class of $G$ has one or two edges. As $G$ has precisely three removable classes, $H$ has at most six edges. It follows that $|V G|=|V H| \leqslant 6$.

By Theorem 1, $G$ has a conformal subgraph which is an even subdivision of $K_{4}$ or $\overline{C_{6}}$. By Lemma 10 and Proposition 11, $G$ is a cubic simple graph. Thus, $G$ must in fact be $K_{4}$ or $\overline{C_{6}}$. But, $\overline{C_{6}}$ is not solid. Therefore, $G$ is $K_{4}$.

\section{Extremal graphs which have a separating cut}

The following theorem, together with Theorem 15, provide an iterative procedure to construct all extremal graphs.

Theorem 16. Let $G$ be a cubic extremal graph and let $C$ be a separating cut of $G$. Then both $C$-contractions are cubic extremal graphs.

Proof: By induction on $|V G|$. We may assume that $C$ is nontrivial, as the conclusion is clear otherwise. By Proposition 11, $G$ is simple. Let $G_{1}$ and $G_{2}$ be the two $C$-contractions. Then $G_{1}$ and $G_{2}$ are matching covered graphs. Every matching covered graph is 2connected. So, $|C| \geqslant 2$. As $G$ is cubic, we must in fact have $|C| \geqslant 3$ (otherwise $G_{1}$ and $G_{2}$ would be graphs with an odd number of vertices of odd degree).

Case 1. $G_{1}$ or $G_{2}$ has no nontrivial separating cut.

Assume, without loss of generality, that $G_{2}$ has no nontrivial separating cut. As every tight cut is a separating cut, $G_{2}$ has no nontrivial tight cut, that is, $G_{2}$ is a brick or brace.

Suppose that $G_{2}$ is a brace. If $\left|V G_{2}\right| \geqslant 6$ then it follows from Lemma 12 that $G_{2}$ has at least six removable edges not in $C$. These edges constitute at least six removable 
classes in $G$. We may thus assume that $\left|V G_{2}\right|=4$. But each vertex of $G_{2}$ is of degree at least 3 , in contradiction to the fact that $G_{2}$ is a simple brace. We conclude that $G_{2}$ must be a brick.

Let $Q_{1}, Q_{2}, \ldots, Q_{k}$ be the removable classes of $G_{1}$. Then, $G_{1}-Q_{i}$ is matching covered, for $i=1,2, \ldots, k$. By Corollary $7, k \geqslant 3$.

If some $Q_{i}$ does not contain an edge of $C$ then $G-Q_{i}$ is matching covered, that is, $Q_{i}$ is a removable class of $G$. Suppose that a removable class $Q_{i}$ of $G_{1}$ contains an edge $e$ of $C$. Let $R_{i}$ be a minimal class of $G_{2}$ induced by $e$, and let $f$ be any edge of $E G_{2}-R_{i}$. Then $f$ does not depend on an edge of the minimal class $R_{i}$, and so $G_{2}-R_{i}$ has a 1-factor containing $f$. Moreover it is connected, by Theorem 8 , since its minimum degree is at least 3. Consequently $G_{2}-R_{i}$ is matching covered. If $R_{i}$ does not contain an edge of $C$ then $G-R_{i}$ is matching covered, so that $R_{i}$ includes a removable class of $G$. In the remaining case, $R_{i} \cap C=\{e\}$. Then $G-\left(Q_{i} \cup R_{i}\right)$ is matching covered, by Proposition 3 . Therefore $Q_{i} \cup R_{i}$ includes a removable class of $G$. Thus, every removable class $Q_{i}$ of $G_{1}$ yields at least one removable class of $G$. The resulting removable classes of $G$ are disjoint since minimal classes of $G_{2}$ induced by distinct edges of $C$ are disjoint. But $G$ has precisely three removable classes. Thus, $G_{1}$ has precisely three removable classes. As asserted, $G_{1}$ is extremal. Thus, $G_{1}$ is cubic, since $|C| \geqslant 3$.

Let us now show that $G_{2}$ is extremal. If $G_{1}$ has no nontrivial separating cut then $G_{2}$ is an extremal graph by the argument above. We may thus assume that $G_{1}$ has a nontrivial separating cut.

Let $D=\partial(Y)$ be a nontrivial separating cut of $G_{1}$ such that the shore, say $Y$, that does not contain the contraction vertex of $G_{1}$ is minimal. Note that every separating cut of $G_{1}$ is also a separating cut of $G$. Then, $D$ is a separating cut of $G$. Let $H_{1}$ and $H_{2}$ be the two $D$-contractions of $G$. Then $H_{1}$ and $H_{2}$ are matching covered graphs. Fix notation such that $\mathrm{H}_{2}$ is obtained by contracting $Y$, so that $C$ is a separating cut of $H_{2}$. By the choice of $D$, graph $H_{1}$ has no nontrivial separating cut. By the argument above, $H_{2}$ is cubic and extremal. As $C$ is a separating cut of $H_{2}$, the inductive hypothesis shows that both $C$-contractions of $H_{2}$ are extremal graphs. One of the $C$-contractions of $H_{2}$ is $G_{2}$. Thus, $G_{2}$ is extremal.

Case 2. $G_{1}$ and $G_{2}$ both have nontrivial separating cuts.

Let $D=\partial(Y)$ be a nontrivial separating cut of $G_{1}$ such that the shore $Y$ that does not contain the contraction vertex of $G_{1}$ is minimal. Then $D$ is also a separating cut of $G$. Let $H_{1}$ and $H_{2}$ be the two $D$-contractions of $G$. Then $H_{1}$ and $H_{2}$ are matching covered graphs. Fix notation such that $H_{2}$ is obtained by contracting $Y$. By Case $1, H_{1}$ and $H_{2}$ are extremal. But $C$ is a separating cut of $H_{2}$. By the inductive hypothesis, both $C$-contractions of $\mathrm{H}_{2}$ are extremal graphs. One of the $C$-contractions of $\mathrm{H}_{2}$ is $G_{2}$. Thus, $G_{2}$ is extremal. Analogously, $G_{1}$ is extremal. By Lemma 10, $G_{1}$ and $G_{2}$ are cubic. 


\section{The list of extremal graphs}

Now, we present a list of the nonbipartite cubic extremal graphs: there are nine of them. The following result is useful to guarantee that every extremal graph is a brick.

Lemma 17. ([6, Corollary 2.8]) Any splicing of two cubic bricks is a (cubic) brick.

By Theorems 15 and 16, every cubic extremal graph distinct from $\Theta$ is obtained by iterative splicings of $K_{4}$. As $K_{4}$ is a cubic brick, it follows from the above lemma that every nonbipartite extremal graph is a brick. As bricks are 3-connected graphs, every minimal class is a removable class, by Theorem 8 .

We shall make use of the following arguments to ensure that a cubic graph is not extremal. The next result is a consequence of the definition of minimal classes.

Proposition 18. Let $G$ be a cubic extremal graph. Then each removable class lies in precisely one perfect matching of $G$.

Proof: Let $v \in V G$. Let $e_{1}, e_{2}$ and $e_{3}$ be the three edges incident on $v$. For $i=1,2,3$, let $Q_{i}$ be a minimal class of $G$ induced by $e_{i}$. As $G$ is extremal, $Q_{1}, Q_{2}$ and $Q_{3}$ are the three removable classes of $G$. By the definition of an induced minimal class, every perfect matching that includes $Q_{i}$ also contains edge $e_{i}$. This assertion holds for any vertex of $G$. Thus, for each $i$ and each vertex $w$ there exists a unique edge that is incident on $w$ and belongs to every perfect matching that includes $Q_{i}$. We conclude that each class $Q_{i}$ lies in a unique perfect matching of $G$.

Let $H$ be a cubic graph and let $v \in V H$. We shall denote by $\left(H \odot K_{4}\right)_{v}$ the graph obtained by splicing $H$ and $K_{4}$ at vertex $v$.

Corollary 19. Let $H$ be a cubic extremal graph and let $v \in V H$. Let $Q$ be a removable class of $H$ which has no edge incident with $v$. Let

$$
G=\left(H \odot K_{4}\right)_{v} .
$$

If there is a perfect matching $M$ in $G$ containing $Q$ and the three edges incident with $v$ then $G$ is not extremal.

Proof: By hypothesis, $H-Q$ is matching covered, and $Q$ has no edge incident with $v$. It follows that $Q$ is a removable class of $G$. But, $Q$ lies in two perfect matchings of $G$, namely, $M$ and the perfect matching of $G$ whose restriction to $E H$ is the perfect matching of $H$ that includes $Q$. By Proposition 18, $G$ is not extremal.

The above corollary suggests the following definition. A vertex $v$ of a cubic graph $H$ is said to satisfy the extension condition if the graph obtained from $H$ by deleting $v$ and all the three neighbours of $v$ does not have a perfect matching containing a removable class of $H$.

The following theorem is an immediate consequence of Theorem 15 and Theorem 16. 
Theorem 20. Let $G$ be a cubic extremal graph different from $K_{4}$. Then

$$
G=\left(H \odot K_{4}\right)_{v},
$$

where $H$ is a cubic extremal graph on $|V(G)|-2$ vertices and $v$ is a vertex of $H$ that satisfies the extension condition.

Theorem 20 suggests how all cubic extremal graphs distinct from $\Theta$ may be generated. We start with $K_{4}$, the only cubic extremal graph on four vertices. For $n \geqslant 4$, suppose that the set $\mathcal{G}_{n}$ of all extremal cubic graphs on $n$ vertices is known. Then each graph in the set $\mathcal{G}_{n+2}$, the set of cubic extremal graphs on $n+2$ vertices, is of the form $\left(H \odot K_{4}\right)_{v}$, where $H$ is a member of $\mathcal{G}_{n}$ and $v$ is a vertex of $H$ that satisfies the extension condition. All graphs (up to isomorphism) that can be generated in this way are shown in Figure 1.

The three removable classes of each extremal graph are indicated in the figure with numbers 1, 2 and 3. Vertices that satisfy the extension condition are labelled $u$ and $v$. Up to automorphisms, no other vertex satisfies the extension condition.

There is only one extremal cubic brick on sixteen vertices, namely $G_{9}$ (Figure 1) and no vertex of this graph satisfies the extension condition. Therefore, there are no cubic extremal graphs on eighteen vertices. It is quite interesting that this procedure cannot be carried on forever.

Corollary 21. Any non-planar matching covered graph has at least four removable classes.

\section{Application}

Let $\mathcal{F}$ be the set of the nine graphs in Figure 1. The next result generalizes the theorem [8] that any two edges of a matching covered graph lie in a conformal circuit.

Corollary 22. Let $G$ be a matching covered graph and let $S$ be a set of three edges of $G$. Then $S$ is contained in a conformal subgraph of $G$ which is induced by a circuit or is an even subdivision of $\Theta$ or of a member of $\mathcal{F}$.

Proof: We use induction on $|E G|$. The corollary holds if $G$ is induced by a circuit, and so we suppose it is not.

Suppose that $G$ contains a removable class $Q$ such that $Q \cap S=\emptyset$. Then $G-Q$ is a conformal matching covered subgraph of $G$ containing $S$. If $G-Q$ is induced by a circuit then we are done. Otherwise the inductive hypothesis shows that $S$ is included in a conformal subgraph $H$ of $G-Q$ which is induced by a circuit or is an even subdivision of $\Theta$ or of a member of $\mathcal{F}$. The conformal property is transitive. Thus, $H$ is a conformal subgraph of $G$.

We may thus assume that every removable class of $G$ contains an edge of $S$. By Corollary 7, $G$ has at least three removable classes. By Lemma 6, the removable classes of $G$ are disjoint. As $|S|=3$, it follows that $G$ has precisely three removable classes, that is, $G$ is extremal. Thus, $G$ is an even subdivision of $\Theta$ or of a member of $\mathcal{F}$. 

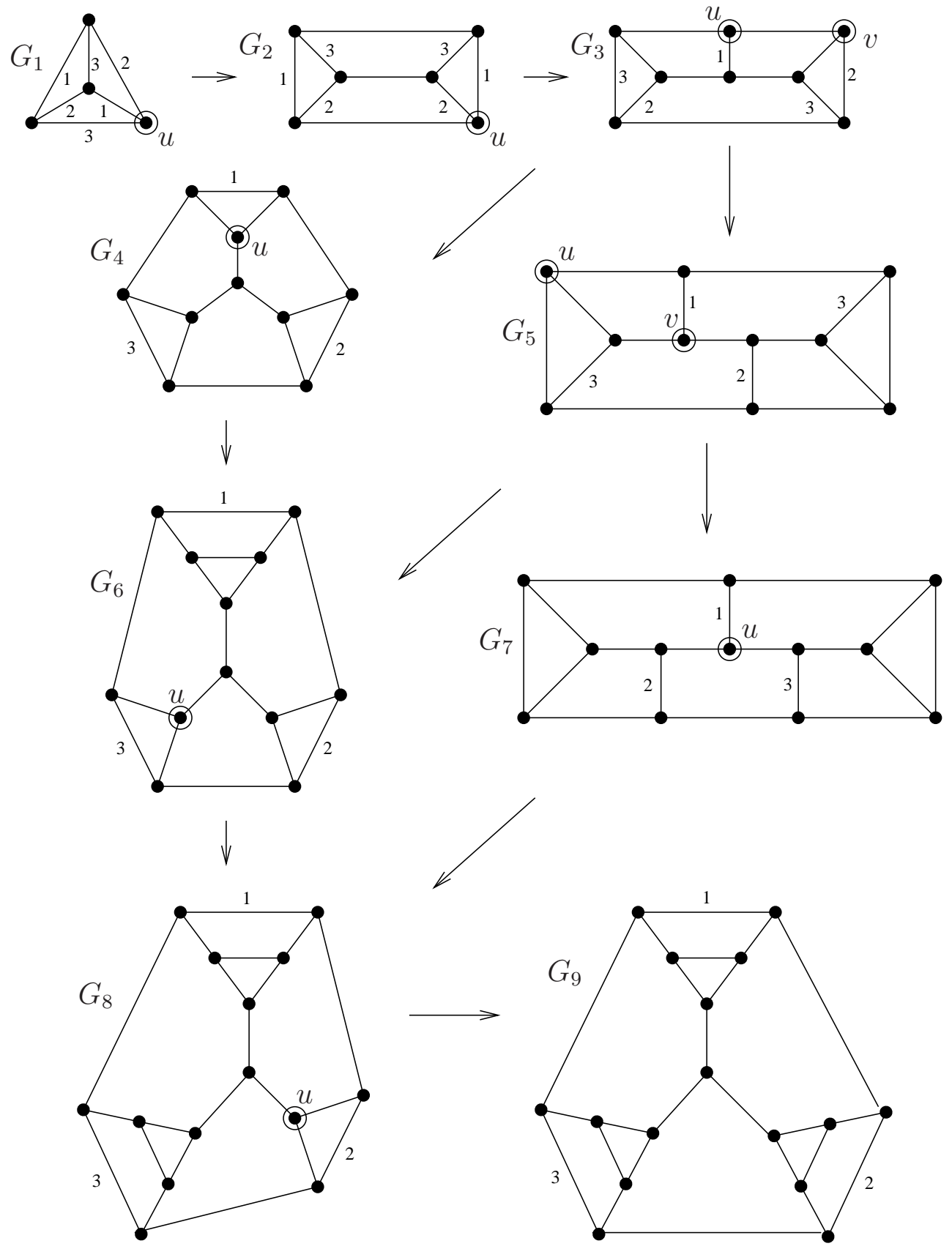

Figure 1: Cubic extremal graphs

\section{References}

[1] J. A. Bondy and U. S. R. Murty. Graph Theory. Springer, 2008.

[2] M. H. Carvalho and C. H. C. Little. Circuit decompositions in join covered graphs. Journal of Graph Theory, 62:220 - 233, 2009. 
[3] M. H. Carvalho. Decomposicao Ótima em Orelhas Para Grafos Matching Covered, Ph.D. thesis, University of Campinas, Brasil, 1986. In Portuguese.

[4] M. H. Carvalho, C. L. Lucchesi and U. S. R. Murty. A Generalization of Little's Theorem on Pfaffian Orientations. Journal of Combinatorial Theory, Series B, 102:12411266, 2012.

[5] M. H. Carvalho, C. L. Lucchesi and U. S. R. Murty. Ear decompositions of matching covered graphs. Combinatorica, 19:151-174, 1999.

[6] M. H. Carvalho, C. L. Lucchesi and U. S. R. Murty. Graphs with independent perfect matchings. Journal of Graph Theory, 48:19-50, 2005.

[7] M. H. Carvalho, C. L. Lucchesi and U. S. R. Murty. The perfect matching polytope and solid bricks. Journal of Combinatorial Theory, Series B, 92:319-324, 2004.

[8] C. H. C. Little. A theorem on connected graphs in which every edge belongs to a 1-factor. J. Austral. Math. Soc., 18:450-452, 1974.

[9] L. Lovász. Ear decompositions of matching covered graphs. Combinatorica, 3:105$117,1983$.

[10] L. Lovász. Matching structure and the matching lattice. Journal of Combinatorial Theory, Series B, 43:187-222, 1987.

[11] L. Lovász and M. D. Plummer. Matching Theory. North-Holland, 1986. 